\title{
Investigating formation of isolated intermediate/massive YSOs in the LMC
}

\author{
Ryohei Harada $^{1}$, Toshikazu Onishi ${ }^{1}$, Annie Hughes ${ }^{8}$, Margaret \\ Meixner $^{2}$, Marta Sewilo ${ }^{9}$, Remy Indebetouw ${ }^{3}$, Omnarayani Nayak ${ }^{10}$, \\ Kazuki Tokuda ${ }^{1}$, Yuuki Morioka ${ }^{1}$, Yasuo Fukui ${ }^{4}$, Akiko Kawamura ${ }^{5}$, \\ Tony Wong ${ }^{6}$, and Jean-Philippe Bernard ${ }^{7}$ \\ ${ }^{1}$ Department of Physical Science, Graduate School of Science, Osaka Prefecture University, \\ 1-1 Gakuen-cho, Naka-ku, Sakai, Osaka 599-8531, Japan \\ email: s_r.harada@p.s.osakafu-u.ac.jp \\ ${ }^{2}$ Space Telescope Science Institute, 3700 San Martin Drive, Baltimore, MD 21218, USA \\ ${ }^{3}$ Department of Astronomy, University of Virginia, P.O. Box 3818, Charlottesville, VA \\ 22903-0818, USA, \\ ${ }^{4}$ Department of Astrophysics, Nagoya University, Chikusa-ku, Nagoya 464-8602, Japan \\ ${ }^{5}$ National Astronomical Observatory of Japan, Mitaka, Tokyo 181-8588, Japan, \\ ${ }^{6}$ Astronomy Department, University of Illinois, Urbana, IL 61801, USA \\ ${ }^{7}$ Université de Toulouse, UPS, CESR, 9 avenue du colonel Roche, 31028 Toulouse Cedex 4, \\ France \\ ${ }^{8}$ Max Planck Institute for Radio Astronomy, Auf dem Huegel 69, Bonn 53121, Germany \\ ${ }^{9}$ Space Science Institute, 4750 Walnut Street, Suite 205, Boulder, CO 80301, USA \\ ${ }^{10}$ The Johns Hopkins University, Department of Physics and Astronomy, 366 Bloomberg \\ Center, 3400 N. Charles Street, Baltimore, MD 21218, USA
}

\begin{abstract}
High-mass stars usually form in giant molecular clouds (GMCs) as part of a young stellar cluster, but some isolated O/B stars are observed. What are the initial conditions that lead to the formation of these objects? The aim of this study is to measure the distribution and basic physical properties of the neutral gas associated with isolated intermediate- and high-mass young stellar objects (YSOs) in the Large Magellanic Cloud.

As part of the SAGE Spitzer Legacy program for the LMC, we have identified and confirmed YSOs using Spitzer IRAC photometry and IRS spectroscopy. By examining the spatial coincidence between the YSOs and ${ }^{12} \mathrm{CO}(1-0)$ emission detected by the NANTEN mapping survey, we identified more than one hundred intermediate/massive YSOs in the LMC that appear to be isolated, i.e. not associated with CO emission. Deeper follow-up CO observations by our team with the higher resolution by Mopra Telescope (beam $=30^{\prime \prime}$ ) detected CO emission at the YSO positions for $\sim 80 \%$ of the isolated LMC YSOs. We obtained ALMA data of some of the targets during Cycle 2. We targeted a small but representative (in terms of their association with neutral gas tracers) sample of the isolated high-mass YSOs that we have been studying in the LMC. All of our 12 targets are separated by more than 200 pc from known CO clouds. Our analysis of the ALMA data shows that a compact molecular cloud whose mass is a few thousand solar masses or smaller is associated with most of the YSOs.
\end{abstract}

Keywords. ISM: clouds, stars: formation, radio lines: ISM

\section{Introduction}

Due to its proximity $(\sim 50 \mathrm{kpc})$, high Galactic latitude $\left(\mathrm{b} \sim-69^{\circ}\right)$ and face-on orientation (i $\sim 35^{\circ}$ ), the LMC is the best place to investigate the origin of isolated high-mass YSOs, as it is there that we have the clearest view of the distribution of molecular clouds and young stars in any galaxy, including our own. 
As part of the Surveying the Agents of a Galaxy's Evolution (SAGE) Spitzer Legacy program for the LMC (SAGE; Meixner et al.(2008)), their team has cataloged 1800 unique YSO candidates in the LMC (Whitney et al.(2008); see also Gruendl \& Chu et al. (2009). By examining the spatial coincidence between the YSOs and ${ }^{12} \mathrm{CO}(1-0)$ emission detected by the NANTEN mapping survey (FWHM beam $=2^{\prime} .6$, Fukui et al. $(2008)$ ), we identified intermediate $/$ massive YSOs in the LMC that appear to be isolated, i.e. not associated with CO emission. All of our targets are separated by more than 200 pc from known CO clouds to exclude potential "runaway" sources. This minimum spatial separation is determined by assuming a maximum speed of $200 \mathrm{~km} / \mathrm{s}$ for a runaway YSO (e.g., Perets \& Šubr 2012) and a conservative high-mass YSO lifetime of $10^{6}$ yrs. Deeper follow-up CO observations by our team with the higher resolution by Mopra Telescope (beam $=30$ ") detected CO emission at the YSO positions for $\sim 80 \%$ of the isolated LMC YSOs. The CO emission associated with the YSOs was sometimes spatially extended, but in some cases, the $\mathrm{CO}$ emission was only detected exactly coincident with the YSO position. The molecular clouds associated with these YSOs must therefore have sizes smaller than $8 \mathrm{pc}$ and masses less than a few thousand solar masses.

\section{Results}

The ALMA observations detected compact clouds toward the 10 isolated YSOs (Fig. 1). Typical cloud radius and mass are a few pc and $\sim 10^{3} M_{\odot}$, respectively. This result confirmed that most of our selected YSOs are just formed or being formed in a compact molecular cloud in an isolated environment, and ALMA spatially resolved the gas distribution. In spite of the fact that the compact clouds are even less massive than Taurus molecular cloud, the star formation activity therein is high. Our analysis indicates that the velocity distribution seems not to be always quiet; the clouds are rather turbulent. The typical linewidth is $4-10 \mathrm{~km} / \mathrm{s}$. There is a possibility that the turbulent motion of the gas may be enhancing the star formation activity. We have discovered molecular outflow for one of the targets that have a velocity span of $10 \mathrm{~km} / \mathrm{s}$ in ${ }^{12} \mathrm{CO}(\mathrm{J}=2-1)$.
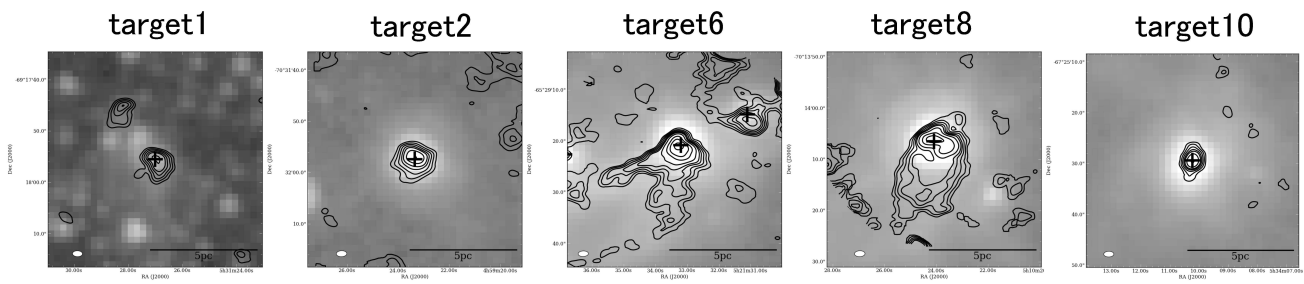

Figure 1. Black contours show ${ }^{12} \mathrm{CO}(2-1)$ integrated intensity taken by ALMA. Gray scale image shows Spitzer $8 \mu \mathrm{m}$. Crosses are positions of high-intermediate mass YSOs. The angular resolution is indicated at the lower-left corner.

\section{References}

Meixner, M.,Gordon, K., Indebetouw, R., et al. 2008, PASA, 25, 149

Whitney, B. A., Sewilo, M., Indebetouw, R., et al 2008, $A J, 136,18$

Gruendl, R. A., \& Chu, Y.-H. 2009, ApJS, 184, 172

Fukui, Y., Kawamura, A., Minamidani, T., et al. 2008, ApJS, 178, 56

Perets, H. B., \& Šubr, L. 2012, ApJ, 751, 133 